\title{
3 Research Square

\section{Potential habitat and productivity loss of Populus deltoides industrial forest plantations due to global warming}

Jiejie Sun

Nanjing Forestry University

Wenxing Jiao

Nanjing Forestry University

Qian Wang

Nanjing Forestry University

Tongli Wang

The University of British Columbia

Hongqiang Yang

Nanjing Forestry University

Jiaxin Jin

Hohai University

Huili Feng

Nanjing Forestry University

Jiahuan Guo

Nanjing Forestry University

Lei Feng

Nanjing Forestry University

Xia Xu

Nanjing Forestry University

Weifeng Wang ( $\nabla$ wang.weifeng@njfu.edu.cn )

Nanjing Forestry University https://orcid.org/0000-0002-9752-6185

Keywords:

Posted Date: May 4th, 2021

DOl: https://doi.org/10.21203/rs.3.rs-438986/v1

License: (c) (i) This work is licensed under a Creative Commons Attribution 4.0 International License.

Read Full License 


\section{Abstract}

The authors have requested that this preprint be removed from Research Square. 